\title{
Enabling Rapid End-to-End Programming of Mobile Manipulators
}

\author{
Justin Huang \\ Computer Science \& Engineering, University of Washington \\ Seattle, WA 98195 \\ jstn@cs.washington.edu
}

\begin{abstract}
Programming mobile manipulator robots requires specialized knowledge of robot perception, manipulation, and software frameworks. This puts robot programming out of reach for programmers who have not invested the time to learn these skills. The goal of our research is to make it possible for all programmers to develop applications for mobile manipulators such as clearing a table, fetching items, or playing games with people. This extended abstract describes our current work on Code3, a framework that all programmers can use to program robots. A user study we conducted showed that non-roboticist programmers were able to use our system to program a PR2 robot to clear a table and retrieve snacks. We also discuss our plans to extend this system to make it more useful and usable for real-world use cases.
\end{abstract}

\section{INTRODUCTION}

Programming mobile manipulator robots requires not just programming skills, it also requires specialized knowledge of robot perception, manipulation, and software frameworks. For a software developer learning to program a robot to perform a mobile manipulation task like fetching a snack, it can take at least a week to set up a development environment and learn software libraries like the Robot Operating System (ROS) [8, 10]. Then, they need to learn to write perception and manipulation code to find and pick the snack and finally deliver it to the user. For someone with no experience programming robots, this might take weeks to accomplish. Even professional roboticists took five days to program the PR2 to fetch a beer from a refrigerator [1]. This puts robot programming out of reach for many people who know how to program but don't have specialized knowledge of robotics. But, if robot programming were accessible to all programmers, we might see more robot applications being developed, field-tested, and adopted.

In our research, we are investigating how non-roboticist programmers can program manipulation tasks on robots in a matter of hours, not weeks. We have developed a framework, Code3, which provides user-friendly interfaces to specify common robot perception and manipulation tasks. We demonstrates how Code 3 can be used to program a wide variety of tasks. We also describe a user

Permission to make digital or hard copies of part or all of this work for personal or classroom use is granted without fee provided that copies are not made or distributed for profit or commercial advantage and that copies bear this notice and the full citation on the first page. Copyrights for third-party components of this work must be honored. For all other uses, contact the owner/author(s).

HRI' 17 Companion March 06-09, 2017, Vienna, Austria

Copyright held by the owner/author(s).

ACM ISBN 978-1-4503-4885-0/17/03

DOI http://dx.doi.org/10.1145/3029798.3034804

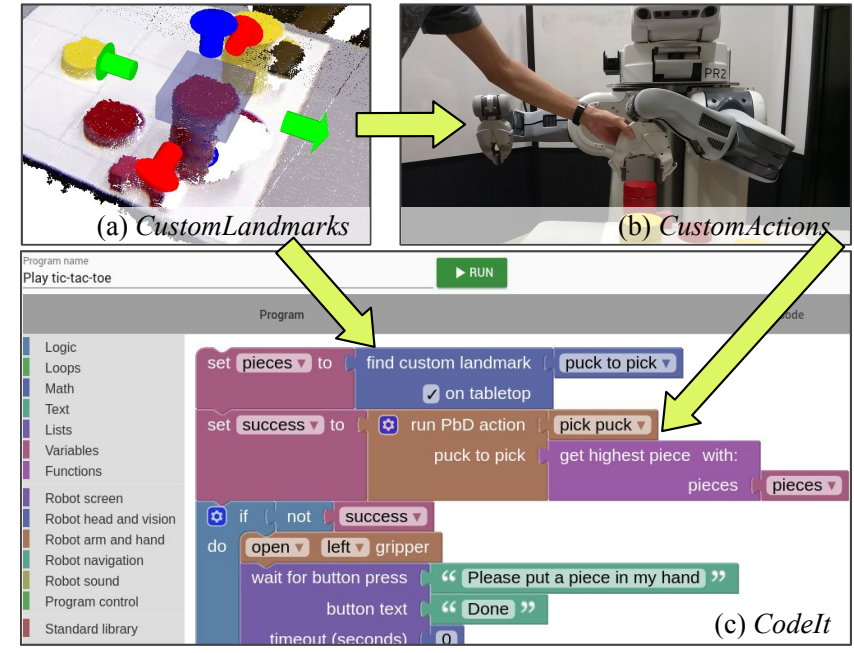

Figure 1: Overview of the Code3 system.

study of ten participants in which users with no robotics experience learned to use our system and programmed the PR2 to do useful tasks in just 2.5 hours. Finally, we discuss our plans to extend the system and make it more useful and usable for real-world use cases.

\section{THE CODE3 FRAMEWORK}

The Code3 framework consists of three tightly-integrated components: CustomLandmarks, CustomActions, and CodeIt.

CustomLandmarks is a user-friendly perception system that lets users define objects or scene parts (called landmarks) that the robot should recognize. To do so, users create a 3D model of the landmark by drawing a box around it in a visualization of the robot's RGBD data, shown in Fig. 11a). Landmarks are detected in a new scene by randomly sampling points in the scene and attempting to align the model to the scene using the Iterative Closest Point algorithm [4]. Users can also model nearby space that is expected to be empty, allowing them to create specific, possibly unusual landmarks that are relevant to their tasks. Detailed algorithms and internal evaluations of the system are described in [6].

CustomActions is a modified version of the keyframe-based programming by demonstration $(\mathrm{PbD})$ system described in [2]. This system allows users to specify manipulation actions by guiding the robot's arms through a sequence of gripper poses, as shown in Fig. 1 b). CustomActions differs from previous work in that it can define actions that are aware of custom landmarks, rather than just tabletop objects. This allows users to create a wider variety of actions, including those that work in non-tabletop settings. 
CodeIt is a programming interface with a high-level API that controls the robot, such as playing sounds or asking questions on a touch screen. It also can be used to locate landmarks or execute manipulation actions created with the two components above. It uses visual editor (Fig.11.c)) in which blocks, representing programming language snippets like loops and if statements, are placed together to form a program. The editor runs on the web so users can log in and program the robot without installing ROS or any tools.

CodeIt is an open-source version of the system described in [7]. We extended their system, which only worked with a mobile robot with no arms, so it could program manipulation actions. Other researchers have developed programming systems for the PR2, including ROSCo [9] and RoboFlow [3]. However, these systems represent control flow with state machines, which are less expressive than the general-purpose programming language provided by CodeIt. For example, the tic-tac-toe program described below would be difficult to develop without a general-purpose language.

\section{PROOF OF CONCEPT DEMOS}

We demonstrated the expressivity of Code 3 by using it to program two complex tasks on the PR2.

In a class at the University of Washington, a group of senior computer science undergraduates spent 10 weeks learning to program the PR2 and programming it to play tic-tac-toe against a human opponent. In contrast, this program took the author 3 hours and 23 minutes to program using Code 3 working alone. To do so, we created a custom landmark of a game piece and programmed a custom action to pick the top game piece from a stack (Fig. 1). We programmed nine custom actions to place the piece in each of the nine squares on the board. We also placed a uniquely-shaped block in the corner of the board, which we made into a custom landmark. The robot located the block and compared it to the locations of game pieces to figure out the board state and decide on its move.

We also programmed the PR2 to use a tool to pick an otherwise ungraspable object out of shelf. The robot located the object and pulled it out with the tool so that the object hung over the shelf edge slightly, where it could be grasped from the front. However, the robot could not pull too little or too much, or else the object would stay unreachable or fall out of the shelf. To address this issue, we created a landmark of the shelf edge, which helped the robot know how far back to pull the object.

\section{USER STUDY}

We conducted a user study of Code 3 with ten non-roboticist programmers. Each user received 60 minutes of in-person training from a standardized script and worked on a familiarization task for 30 minutes. Then, they were given 50 minutes to program one of two tasks. One task was to ask the user which of two snacks they wanted, and to have the robot recognize the appropriate snack, pick it up, and hand it to the user. The other task was to clear a table of bowls and carry the bowls away. All ten users were able to program one of the tasks, and three users had extra time to program the other task. The mean time to program a task was 31:38 minutes. Improving on the system's usability is an area for future work. For example, users encountered issues with their actions that needed to be tweaked and incrementally edited, which we did not support. Overall, users rated the system a 66.75 on average on the System Usability Scale [5], which is considered slightly below average.

\section{CONCLUSION AND FUTURE WORK}

Our goal is to make robot programming accessible to all programmers, which could allow many more people to explore new uses for robots. We developed the Code 3 framework, which allows general programmers to build their own object detectors with $\mathrm{Cus}$ tomLandmarks, specify manipulation actions that act on landmarks with CustomActions, and to program the robot to execute actions using CodeIt. We demonstrated that the system is highly expressive by programming tasks like playing tic-tac-toe with a human player. Importantly, we showed that programmers without robotics experience were able to use Code 3 to program the robot to do useful tasks like fetching a snack or clearing a table.

In future work, we want to apply the system to real-world settings like picking and stowing items in grocery stores. This will require improvements in design and technical capabilities. The system needs an improved editor that helps users incrementally edit $\mathrm{PbD}$ actions. To execute precise movements, we also need to develop ways for users to specify compliant motions on the robot, e.g., stopping a pushing action when a certain amount of force is felt. Finally, the perception system could be more flexible by using $2 \mathrm{D}$ color information in addition to 3D shape. Through this work, we hope to develop a robust, flexible system that all programmers can use to discover new uses for mobile manipulator robots.

\section{Acknowledgments}

This work was supported by the National Science Foundation, Awards IIS-1552427 "CAREER: End-User Programming of General-Purpose Robots" and IIS-1525251 "NRI: Rich Task Perception for Programming by Demonstration."

\section{REFERENCES}

[1] Beer me, robot. Willow Garage Inc. Blog. http: //www.willowgarage.com/blog/2010/07/06/beer-me-robot July 2010.

[2] S. Alexandrova, M. Cakmak, K. Hsiao, and L. Takayama. Robot programming by demonstration with interactive action visualizations. In Robotics: Science and Systems (RSS), pages 48-56, 2014.

[3] S. Alexandrova, Z. Tatlock, and M. Cakmak. RoboFlow: A flow-based visual programming language for mobile manipulation tasks. In International Conference on Robotics and Automation. IEEE, 2015.

[4] P. J. Besl and N. D. McKay. A method for registration of 3-D shapes. IEEE Trans. Pattern Anal. Mach. Intell., 14:239-256, 1992.

[5] J. Brooke et al. SUS-a quick and dirty usability scale. Usability Evaluation in Industry, 189(194):4-7, 1996.

[6] J. Huang and M. Cakmak. Programming by demonstration with user-specified perceptual landmarks. arXiv preprint arXiv:1612.00565, 2016.

[7] J. Huang, T. Lau, and M. Cakmak. Design and evaluation of a rapid programming system for service robots. In 11th ACM/IEEE International Conference on Human-Robot Interaction. IEEE, 2016.

[8] Y. Lin. Learn ROS in a single week. ROS Discourse. http://discourse.ros.org/t/learn-ros-in-a-single-week/680, October 2016.

[9] H. Nguyen, M. Ciocarlie, K. Hsiao, and C. C. Kemp. ROS Commander (ROSCo): Behavior creation for home robots. In Robotics and Automation (ICRA), 2013 IEEE International Conference on. IEEE, 2013.

[10] M. Quigley, K. Conley, B. Gerkey, J. Faust, T. Foote, J. Leibs, R. Wheeler, and A. Y. Ng. ROS: an open-source robot operating system. In ICRA Workshop on Open Source Software. Kobe, Japan, 2009. 